\title{
Probiotic Intervention Through the Pregnant and Breastfeeding Mother to Reduce Disease Risk in the Child
}

\author{
Samuli Rautava
}

$\mathbf{T}$ HE INCIDENCE OF INFECTIOUS diseases has declined drastically since the 1950s, yet the incidence of chronic, noncommunicable diseases has been steadily increasing. ${ }^{1}$ Increased risk of noncommunicable diseases is associated with disturbances in microbial contact and gut microbiota composition during infancy. $^{2-5}$ The mode of delivery (i.e., vaginal birth or cesarean delivery) can greatly impact the initial microbiota in newborns. ${ }^{6}$ Cesarean deliveries are associated with reduced gut microbiota diversity and increased risk of many chronic immune disorders (e.g., asthma, inflammatory bowel diseases) later in life. ${ }^{7-9}$ Breastfeeding is also an important modulator of the gut microbiome and is associated with reduced risk of disease. Human milk contains factors (e.g., human milk oligosaccharides) that promote the growth of beneficial bacteria and may also be a source of bacteria for the developing gut. ${ }^{10}$ The microbial composition of breast milk has been shown to vary with the mode of delivery and can change over the course of lactation. ${ }^{11}$ Thus, modulation of early microbial contact through avoidance of unnecessary cesarean sections, promotion of breastfeeding, and prudent use of antibiotics may improve health outcomes.

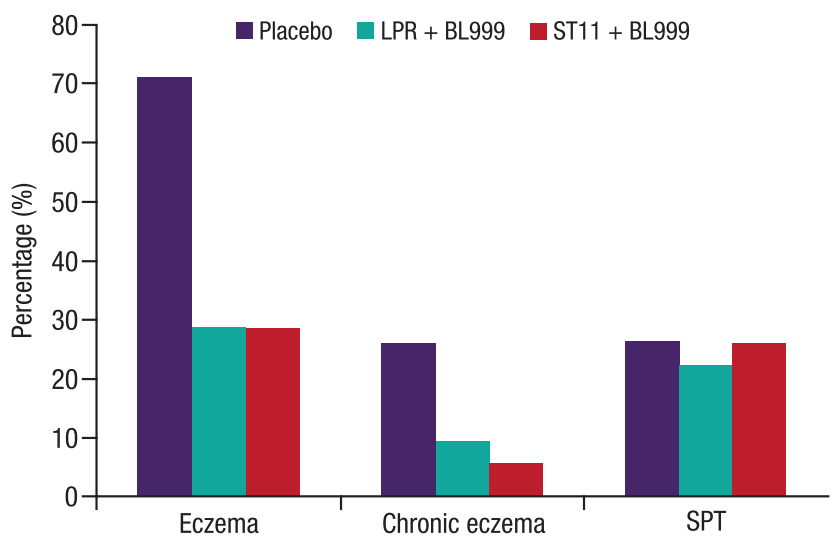

FIG. 1. Maternal probiotic supplementation during pregnancy and breastfeeding reduces the risk of developing eczema in high-risk infants. ${ }^{15}$ BL999, Bifidobacterium longum BL999; LPR, Lactobacillus rhamnosus LPR; SPT, skin prick test; ST11, Lactobacillus paracasei ST11.
Other interventions, such as vaginal seeding after cesarean delivery and the use of prebiotics and probiotics, have also been investigated as a means to influence early microbial contact and to reduce disease risk.

Probiotics are defined as "live micro-organisms that, when administered in adequate amounts, confer a health benefit on the host." ${ }^{12}$ Prenatal probiotic administration to high-risk pregnant women 2-4 weeks before delivery and 6 months postnatally to their infants has been shown to significantly decrease the risk of atopic eczema during the first 7 years of life. ${ }^{13,14}$ Findings from a separate study demonstrated that probiotic supplementation exclusively to the mother (i.e., the infants did not receive probiotics) during the last 2 months of pregnancy and the first 2 months of breastfeeding was also effective in reducing the risk of eczema in high-risk infants during the first 2 years of life (Fig. 1). ${ }^{15}$

Probiotic supplementation in pregnant and breastfeeding mothers may result in the transfer of probiotic bacteria to the infant and/or increases in gut microbiota diversity; however, recent data suggest that maternal probiotic supplementation does not alter the infant microbiota. ${ }^{16}$ The preventive mechanism of probiotic intervention in the mother may be associated with increases in immunomodulatory factors (e.g., cytokines and growth factors) found in breast milk. Higher concentrations of transforming growth factor $\beta 2$ (TGF- $\beta 2$ ) have been reported in the breast milk of mothers receiving probiotics than in the breast milk of mothers receiving placebo. ${ }^{13}$ Furthermore, TGF- $\beta 2$ has been shown to attenuate interleukin $1 \beta$-induced production of proinflammatory cytokines in immature human intestinal epithelial cells. ${ }^{17}$

Probiotic supplementation during pregnancy may modulate prenatal microbial contact and fetal immune physiology. Microbes have been detected in healthy human placenta, amniotic fluid, and meconium. ${ }^{18,19}$ A recent study characterizing these microbial populations demonstrated that the meconium microbiota share features with the microbiota of the placenta and amniotic fluid, suggesting that the initial inoculum for gut colonization during fetal development may originate from the placenta and amniotic fluid. ${ }^{20}$ Consistent with this, probiotic intervention during the last 2 weeks of pregnancy was associated with changes in innate immune gene expression profiles in the placenta and fetal gut. ${ }^{21}$

Neonatologist, Turku University Hospital; and Adjunct Professor of Experimental Pediatrics, University of Turku, Turku, Finland. 
Together, these findings suggest that early microbial contact likely begins in utero and can be affected by various factors, such as mode of delivery and breastfeeding. ${ }^{22}$ Probiotic intervention during pregnancy and breastfeeding is associated with lower risk of disease, which may be due to enhanced protective effects of breast milk and modulation of prenatal microbial contact.

\section{References}

1. Bach JF. The effect of infections on susceptibility to autoimmune and allergic diseases. N Engl J Med 2002;347: 911-920.

2. Kalliomäki M, Kirjavainen $\mathrm{P}$, Eerola E, et al. Distinct patterns of neonatal gut microflora in infants in whom atopy was and was not developing. J Allergy Clin Immunol 2001;107:129-134.

3. Abrahamsson TR, Jakobsson HE, Andersson AF, et al. Low diversity of the gut microbiota in infants with atopic eczema. J Allergy Clin Immunol 2012;129:434-440.

4. Gevers D, Kugathasan S, Denson LA, et al. The treatmentnaive microbiome in new-onset Crohn's disease. Cell Host Microbe 2014;15:382-392.

5. Kalliomäki M, Collado MC, Salminen S, et al. Early differences in fecal microbiota composition in children may predict overweight. Am J Clin Nutr 2008;87:534-538.

6. Dominguez-Bello MG, Costello EK, Contreras M, et al. Delivery mode shapes the acquisition and structure of the initial microbiota across multiple body habitats in newborns. Proc Natl Acad Sci U S A 2010;107:11971-11975.

7. Jakobsson HE, Abrahamsson TR, Jenmalm MC, et al. Decreased gut microbiota diversity, delayed Bacteroidetes colonisation and reduced Th1 responses in infants delivered by caesarean section. Gut 2014;63:559-566.

8. Kumbhare SV, Kumar H, Chowdhury SP, et al. A crosssectional comparative study of gut bacterial community of Indian and Finnish children. Sci Rep 2017;7:10555.

9. Sevelsted A, Stokholm J, Bønnelykke K, et al. Cesarean section and chronic immune disorders. Pediatrics 2015; 135:e92-e98.

10. Drell T, Štšepetova J, Simm J, et al. The influence of different maternal microbial communities on the development of infant gut and oral microbiota. Sci Rep 2017;7:9940.

11. Cabrera-Rubio R, Collado MC, Laitinen K, et al. The human milk microbiome changes over lactation and is shaped by maternal weight and mode of delivery. Am J Clin Nutr 2012;96:544-551.

12. Hill C, Guarner F, Reid G, et al. Expert consensus document. The International Scientific Association for Probio- tics and Prebiotics consensus statement on the scope and appropriate use of the term probiotic. Nat Rev Gastroenterol Hepatol 2014;11:506-514.

13. Rautava S, Kalliomäki M, Isolauri E. Probiotics during pregnancy and breast-feeding might confer immunomodulatory protection against atopic disease in the infant. $J \mathrm{Al}$ lergy Clin Immunol 2002;109:119-121.

14. Kalliomäki M, Salminen S, Poussa T, et al. Probiotics during the first 7 years of life: A cumulative risk reduction of eczema in a randomized, placebo-controlled trial. $\mathrm{J} \mathrm{Al}$ lergy Clin Immunol 2007;119:1019-1021.

15. Rautava S, Kainonen E, Salminen S, et al. Maternal probiotic supplementation during pregnancy and breast-feeding reduces the risk of eczema in the infant. J Allergy Clin Immunol 2012;130:1355-1360.

16. Dotterud CK, Avershina E, Sekelja M, et al. Does maternal perinatal probiotic supplementation alter the intestinal microbiota of mother and child? J Pediatr Gastroenterol Nutr 2015;61:200-207.

17. Rautava S, Nanthakumar NN, Dubert-Ferrandon A, et al. Breast milk-transforming growth factor- $\beta_{2}$ specifically attenuates IL- $1 \beta$-induced inflammatory responses in the immature human intestine via an SMAD6- and ERK-dependent mechanism. Neonatology 2011;99:192-201.

18. Ardissone AN, de la Cruz DM, Davis-Richardson AG, et al. Meconium microbiome analysis identifies bacteria correlated with premature birth. PLoS One 2014;9:e90784.

19. Aagaard K, Ma J, Antony KM, et al. The placenta harbors a unique microbiome. Sci Transl Med 2014;6:237ra65.

20. Collado MC, Rautava S, Aakko J, et al. Human gut colonisation may be initiated in utero by distinct microbial communities in the placenta and amniotic fluid. Sci Rep 2016;6:23129.

21. Rautava S, Collado MC, Salminen S, et al. Probiotics modulate host-microbe interaction in the placenta and fetal gut: A randomized, double-blind, placebo-controlled trial. Neonatology 2012;102:178-184.

22. Rautava S, Luoto R, Salminen S, et al. Microbial contact during pregnancy, intestinal colonization and human disease. Nat Rev Gastroenterol Hepatol 2012;9:565-576.

Address correspondence to: Samuli Rautava, $M D, P h D$

Department of Pediatrics University of Turku Turku 20014 Finland

E-mail: samulirautava@gmail.com 